\title{
Predictor-based stabilization for chained form systems with input time delay
}

\author{
FAÏÇAL MNIF
}

\begin{abstract}
This note addresses the stabilization problem of nonlinear chained-form systems with input time delay. We first employ the so-called $\sigma$-process transformation that renders the feedback system under a linear form. We introduce a particular transformation to convert the original system into a delay-free system. Finally, we apply a state feedback control, which guarantees a quasi-exponential stabilization to all the system states, which in turn converge exponentially to zero. Then we employ the so-called -type control to achieve a quasi-exponential stabilization of the subsequent system. A simulation example illustrated on the model of a wheeled mobile robot is provided to demonstrate the effectiveness of the proposed approach.
\end{abstract}

Key words: $\sigma$-process transformation, chained form systems, mobile robots, exponential stabilization, time delay system.

\section{Introduction}

Within the last three decades the stabilization problem of nonholonomic mechanical systems has occupied a central role in the nonlinear systems literature. Though nonholonomic systems are controllable, they cannot be stabilized by stationary continuous state feedback because they fail to satisfy Brockett's necessary condition [1]. As a result, the well-posed smooth nonlinear control algorithms cannot be directly applied to stabilize such systems. To overcome this obstacle, a set of approaches and new methodologies have been suggested that made the problem of stabilization of nonholonomic system solvable. [2] and [3] and references therein give a good overview of these methods. These methods are within three categories: Smooth time-invariant methods [4], [5], discontinuous time invariant methods [6],7] to cite few which are based on the so-called $\sigma$-process transformation [6], and hybrid stabilization techniques, [8] and references therein.

On the other hand, one of the current trends in control engineering is to establish new strategies that allow remote control of different systems including mobile robots and manipulators [9]. The use of communication networks for connecting robotic sys-

The authors is with Department of Electrical and Computer Engineering, Sultan Qaboos University, P.O. Box 33, Muscat, Oman. E-mail: mnif@squ.edu.om

Received 15.07.2016. Revised 18.10.2016. 
tems with their controllers is leading to substantial advantages such as increased flexibility enhanced portability [10]. However, networking control channels are subjected to unavoidable time delays that do not only degrade the performance of the control system but also can pose impediments to the system stability if they not carefully addressed. Time-delayed systems attracted the attention of many control theorists and practitioners because of the stability challenges they face. Guaranteeing stability to a control system has been always a challenge for control theorists. Several techniques have been proposed in the context of teleoperation to get around the negative effect of the induced time delay (see [11] for an overview of different proposed methods). All these methods have been applied to unconstrained mechanical systems. Few attempts however have however been given to time-delayed nonholonomic system.

This note addresses the problem of the stabilization of a special class of nonholonomic mechanical systems; the chained form systems, when they are subjected to input time delays. In fact, many nonholonomic mechanical systems including mobile robots can be reduced to such a form by an appropriate local or global change of variables. These type of systems are usually linked via delay-induced wireless communication channels, which may compromise the performance and the stability of the controlled system.

To address the stabilization problem of chained-form systems, we apply a linear transformation consisting of an input to state scaling. We then propose a twodimensional control in the form of quasi-linear state feedback that maintains stabilization of the system states and their convergence to the origin with the presence time delay.

The layout of this note is as follows: In next section, we address nonholonomic systems in chained form. Sections 3 and 4 are devoted to the control design firstly, of the non-delayed system, and then the result is extended to the delayed chained form system in section 4. In Section 5 we provide simulation results of the proposed control algorithm on a Wheeled Mobile Robot (WMR).

\section{Nonholonomic systems in chained form}

In general, an n-dimensional chained form writes as

$$
\begin{gathered}
\dot{x}_{1}=u_{1} \\
\dot{x}_{2}=u_{2} \\
\dot{x}_{3}=x_{2} u_{1} \\
\vdots \\
\dot{x}_{n}=x_{n-1} u_{1}
\end{gathered}
$$

where $x=\left(x_{1}, x_{2}, \ldots, x_{n}\right)^{T}$ is the state vector of the system and $u=\left(u_{1}, u_{2}\right)^{T}$ is the control input. 
Various formulations of control schemes have been developed to resolve for the failure of chained form system to satisfy Brockett's condition [1], namely time-dependent controls, a variety of discontinuous control schemes such as sliding mode and backstepping control. In [10] and references therein, authors present an extensive review of such techniques.

\section{Discontinuous control of chained forms}

Applying the $\sigma$-process coordinate transformation [6] to system (1) yields

$$
\begin{gathered}
\xi_{1}=x_{1} \\
\xi_{2}=x_{2} \\
\xi_{3}=\frac{x_{3}}{x_{1}} \\
\vdots \\
\xi_{n-1}=\frac{x_{n-1}}{x_{1}^{n-3}} \\
\xi_{n}=\frac{x_{n}}{x_{1}^{n-2}} .
\end{gathered}
$$

In the new coordinates the system is described by the following set of equations

$$
\begin{gathered}
\dot{\xi}_{1}=v_{1} \\
\dot{\xi}_{2}=v_{2} \\
\dot{\xi}_{3}=\frac{\xi_{2}-\xi_{3}}{\xi_{1}} v_{1} \\
\vdots \\
\dot{\xi}_{n}=\frac{\xi_{n-1}-(n-2) \xi_{n}}{\xi_{1}} v_{1} .
\end{gathered}
$$

Consider for the subsystem- $\xi_{1}$ the control law $v_{1}=-k \xi_{1}=-k x_{1}$, then the resulting system is designated by the state equation $\dot{\xi}=A \xi+B v_{2}$, where

$$
A=\left[\begin{array}{cccccccc}
-k & 0 & 0 & 0 & \ldots & 0 & 0 & 0 \\
0 & 0 & 0 & 0 & \ldots & 0 & 0 & 0 \\
0 & -k & k & 0 & \ldots & 0 & 0 & 0 \\
0 & 0 & -k & 2 k & \ldots & 0 & 0 & 0 \\
\vdots & \vdots & \vdots & \vdots & \ddots & \vdots & \vdots & \vdots \\
0 & 0 & 0 & 0 & 0 & 0 & -k & (n-2) k
\end{array}\right]
$$


and

$$
B^{T}=\left[\begin{array}{lllll}
0 & 1 & 0 & \ldots & 0
\end{array}\right] .
$$

The linear system (3) can be exponentially stabilized using the linear control

$$
v=\left[\begin{array}{l}
v_{1} \\
v_{2}
\end{array}\right]=\left[\begin{array}{c}
-k_{1} \xi_{1} \\
p_{2} \xi_{2}+p_{3} \xi_{3}+\cdots+p_{n} \xi_{n}
\end{array}\right] .
$$

Proposition 1 In the $x$-coordinates the control law expressed as

$$
v=\left[\begin{array}{l}
v_{1} \\
v_{2}
\end{array}\right]=\left[\begin{array}{c}
-k_{1} x_{1} \\
p_{2} x_{2}+p_{3} \frac{x_{3}}{x_{1}}+\cdots+p_{n} \frac{x_{n}}{x_{1}^{n-2}}
\end{array}\right] .
$$

with $k>0$ and the $p_{i}$ are such that the eigenvalues of the matrix $A+B p$ have negative real parts $p=\left[\begin{array}{llll}0 & p_{2} & \cdots & p_{n-1} \\ p_{n}\end{array}\right]$ globally exponentially stabilizes the system (2) in the open dense set $\Omega_{1}:=\left\{x \in \mathfrak{R}^{n}: x_{1} \neq 0\right\}$.

It is possible to show that the discontinuous control law (6) is well defined and bounded for all $t \geqslant 0$, along the trajectories of the closed loop system (3)-(5) whenever $x_{1}(0) \neq 0$.

\section{Time-delayed chained form system}

Consider now the chained-form system with input time delay. The time delay is assumed to be constant and of the same value for both inputs.

$$
\begin{gathered}
\dot{x}_{1}=v_{1}(t-\tau) \\
\dot{x}_{2}=v_{2}(t-\tau) \\
\dot{x}_{3}=x_{2} v_{1}(t-\tau) \\
\vdots \\
\dot{x}_{n}=x_{n-1} v_{1}(t-\tau)
\end{gathered}
$$

where $\operatorname{\tau in} \Re_{+}$is the input time delay.

In the $\xi$-coordinates, the system dynamics writes as

$$
\begin{gathered}
\dot{\xi}_{1}=v_{1}(t-\tau) \\
\dot{\xi}_{2}=v_{2}(t-\tau) \\
\dot{\xi}_{3}=\frac{\xi_{2}-\xi_{3}}{\xi_{1}} v_{1}(t-\tau) \\
\vdots \\
\dot{\xi}_{n}=\frac{\xi_{n-1}-(n-2) * \xi_{n}}{\xi_{1}} v_{1}(t-\tau)
\end{gathered}
$$


The control objective is to find the new input vector $v^{T}=\left[v_{1} v_{2}\right]$ that achieves exponential convergence of the states to the origin.

\subsection{Predictor-based design and the reduction approach}

Consider the following linear system with input time delay

$$
\dot{\xi}=A \xi+B v(t-\tau), \quad \xi \in \Re^{n}, \quad v \in \mathfrak{R}^{m}
$$

and where $\tau$ is a bounded constant time delay. If we apply the transformation

$$
\eta=\xi+\int_{t-\tau}^{\tau} e^{A(t-\tau-\varphi)} B v(\varphi) d \varphi
$$

then the pseudo-linear z-dynamics write as

$$
\dot{\eta}=A \eta+\bar{B} v, \quad \bar{B}=e^{-A \tau} B
$$

Note: It is straightforward to see from the definition of $\bar{B}$ that if the pair $(A, B)$ is controllable then the pair $(A, \bar{B})$ is also controllable.

Proposition 2 If a state feedback $v=-K \eta$ that stabilizes (11) exists, then the system (9) is also exponentially stable.

Proof From the linear transformation (10), we have

$$
\begin{aligned}
&\|\xi(t)\|=\left\|\eta(t)-\int_{t-\tau}^{t} e^{A(t-\tau-\varphi)} B v(\varphi) d \varphi\right\| \\
& \leqslant\|\eta(t)\|+\left\|\int_{t-\tau}^{t} e^{A(t-t a u-\varphi)} B v(\varphi) d \varphi\right\| \\
& \leqslant\|\eta(t)\|+\tau \max _{-\tau \leqslant \varphi \leqslant 0}\left\|e^{A \varphi}\right\|\|B\|\|K\|\|v(t+\varphi)\| \\
& \leqslant\|\eta(t)\|+\tau \max _{-\tau \leqslant \varphi \leqslant 0}\left\|e^{A \varphi}\right\|\|B\|\|K\|\|\eta(t+\varphi)\|
\end{aligned}
$$

Since system (11) is quasi-exponentially stable, it follows that $\lim _{t \rightarrow \infty} \eta(t)=0$, then $\lim _{t \rightarrow \infty} \xi(t)=0$ and the pseudo-linear system (9) is quasi-exponentially stable. 


\subsection{Design of $v_{1}$ for the $\xi_{1}$-subsystem}

For $\xi_{1}$-subsystem we consider the linear transformation

$$
\eta_{1}(t)=\xi_{1}+\int_{t-\tau}^{\tau} e^{t-\tau-\varphi} v_{1}(\varphi) d \varphi
$$

which transforms it into

$$
\dot{\eta}_{1}(t)=e^{-\tau} v_{1}(t)
$$

for which the control input can be chosen as

$$
v_{1}(t)=-k \eta_{1}(t)
$$

where $k$ is as in (5).

Proposition 3 If for any $t_{0} \geqslant \tau$, the corresponding solution $\xi_{1}(t)$ exists and satisfies $\lim _{t \rightarrow \infty} \xi_{1}(t)=0$. Moreover $\xi_{1}(t)=x_{1}(t)$ does not cross the origin for all $t \in\left[t_{0}, \infty\right)$.

Proof Substituting (14) in (13), yields

$$
\eta_{1}(t)=\eta_{1}\left(t_{0}\right) e^{-k e^{-\tau}\left(t-t_{0}\right)}
$$

which obviously converges to zero as $t \rightarrow \infty$. Moreover,

$$
\xi_{1}(t)=\eta_{1}(t)+k \int_{t-\tau}^{\tau} e^{t-\tau-\varphi} \eta_{1}(\varphi) d \varphi
$$

which along with (15) suggests that $\xi_{1}(t)$ exists and satisfies $\lim _{t \rightarrow \infty} \xi_{1}(t)=0$. On the other hand since (15) indicates that $\eta_{1}(t)$ does not cross zero for all $t \in\left[t_{0}, \infty\right)$, and from (14), so it is for $\xi_{1}(t)=x_{1}(t)$.

\subsection{Design of $v_{2}$}

Consider $\dot{\xi}=A \xi+B v_{2}(t-\tau)$ with

$$
A=\left[\begin{array}{cccccccc}
-k & 0 & 0 & 0 & \ldots & 0 & 0 & 0 \\
0 & 0 & 0 & 0 & \ldots & 0 & 0 & 0 \\
0 & -k & k & 0 & \ldots & 0 & 0 & 0 \\
0 & 0 & -k & 2 k & \ldots & 0 & 0 & 0 \\
\vdots & \vdots & \vdots & \vdots & \ddots & \vdots & \vdots & \vdots \\
0 & 0 & 0 & 0 & 0 & 0 & -k & (n-2) k
\end{array}\right]
$$


and

$$
B^{T}=\left[\begin{array}{lllll}
0 & 1 & 0 & \ldots & 0
\end{array}\right]
$$

and consider the transformation

$$
\eta=x+\int_{t-\tau}^{\tau} e^{A(t-\tau-\varphi)} B v_{2}(\varphi) d \varphi
$$

yields

$$
\dot{\eta}=A \eta+\bar{B} v_{2}
$$

where $\bar{B}=e^{-A \tau} B$, the following result follows:

Proposition 4 For the system (8), if the control law $v^{T}=\left[\begin{array}{ll}v_{1} & v_{2}\end{array}\right]$ such that

$$
v=\left[\begin{array}{l}
v_{1} \\
v_{2}
\end{array}\right]=\left[\begin{array}{c}
-k\left\{\xi_{1}+\int_{t-\tau}^{\tau} e^{t-\tau-\varphi} v_{1}(\varphi) d \varphi\right\} \\
{\left[0 p_{2} p_{3} \ldots p_{n}\right]\left\{\begin{array}{c}
\tau \\
\xi+\int_{t-\tau}^{\tau} e^{A(t-\tau-\varphi)} B v_{2}(\varphi) d \varphi
\end{array}\right]}
\end{array}\right]
$$

and $\xi$ is defined as in (2), the delayed closed loop system globally exponentially stabilizes the system (3) in the open dense set $\Omega_{1}:=\left\{\xi \in \mathfrak{R}^{n}: \xi_{1} \neq 0\right\}$, moreover $\xi_{1}(t)=x_{1}(t)$ does not cross the origin for all $t \in[\tau, \infty)$, and by virtue of Proposition 1, the result follows for the original coordinates system.

\section{Simulation example}

Consider the kinematic model of a wheeled mobile robot

$$
\begin{gathered}
\dot{z}=u \cos \theta \\
\dot{y}=u \sin \theta \\
\dot{\theta}=\omega
\end{gathered}
$$

where $(z, y)$ represents the position of the center of the mass of robot, $\theta$ is its heading angle, $u$ is its linear velocity and $\omega$ is its angular velocity.

Apply the global coordinate transformation

$$
\begin{gathered}
x_{1}=\theta \\
x_{2}=z \cos \theta+y \sin \theta \\
x_{3}=z \sin \theta-y \cos \theta
\end{gathered}
$$


and the static state feedback

$$
u=v_{2}+x_{3} v_{1} \omega=v_{1}
$$

we obtain

$$
\begin{gathered}
\dot{x}_{1}=v_{1} \\
\dot{x}_{2}=v_{2} \\
\dot{x}_{3}=x_{2} v_{1} .
\end{gathered}
$$

Considering a constant time delay $\tau$ in both inputs. This time delay may in fact due to data transmission delay or packet-loss in the case of wireless communication channels. We get

$$
\begin{gathered}
\dot{x}_{1}=v_{1}(t-\tau) \\
\dot{x}_{2}=v_{2}(t-\tau) \\
\dot{x}_{3}=x_{2} v_{1}(t-\tau) .
\end{gathered}
$$

In terms of $\xi$-coordinates, the system expresses as

$$
\begin{gathered}
\dot{\xi}_{1}=v_{1}(t-\tau) \\
\dot{\xi}_{2}=v_{2}(t-\tau) \\
\dot{\xi}_{3}=\frac{\xi_{2}-\xi_{3}}{\xi_{1}} v_{1}(t-\tau) .
\end{gathered}
$$

which results in the system described by $\dot{\xi}=A \xi+\bar{B}_{2} v_{2}$ where

$$
A=\left[\begin{array}{ccc}
-k & 0 & 0 \\
0 & 0 & 0 \\
0 & -k & k
\end{array}\right]
$$

and $\bar{B}_{2}=e^{-A \tau}\left[\begin{array}{lll}0 & 1 & 0\end{array}\right]^{T}$. It is clear that (22) is a simple form of (3). Henceforth, the application of the control law is direct. Assume $\tau=0.5 \mathrm{sec}$ and the design gains are chosen such as $k>0$ and so as to set the coefficients $p_{2}$ and $p_{3}$ in the matrix

$$
A=\left[\begin{array}{cc}
p_{2} & p_{3} \\
-k & k
\end{array}\right]
$$

such as $\sigma\left(A_{3}\right) \in C^{-}$. Setting $k=1$, gives $p_{2}=-3$ and $p_{3}=4$.

Fig. 1 shows the response of the system in its original coordinates $\mathrm{x}$ with the initial conditions? $x_{0}=\left[\begin{array}{ll}4.95 & \frac{-\pi}{2}\end{array}\right]^{T}$. It is clear that the original system coordinates converge exponentially to zero. Note also that the first coordinate $x_{1}$ does not cross zero as claimed in proposition 3. The trajectories of the system responses in $\xi$-coordinates are depicted in Fig. 2, whereas Fig. 3 represents the control inputs $v_{1}$ and $v_{2}$ to the system. 


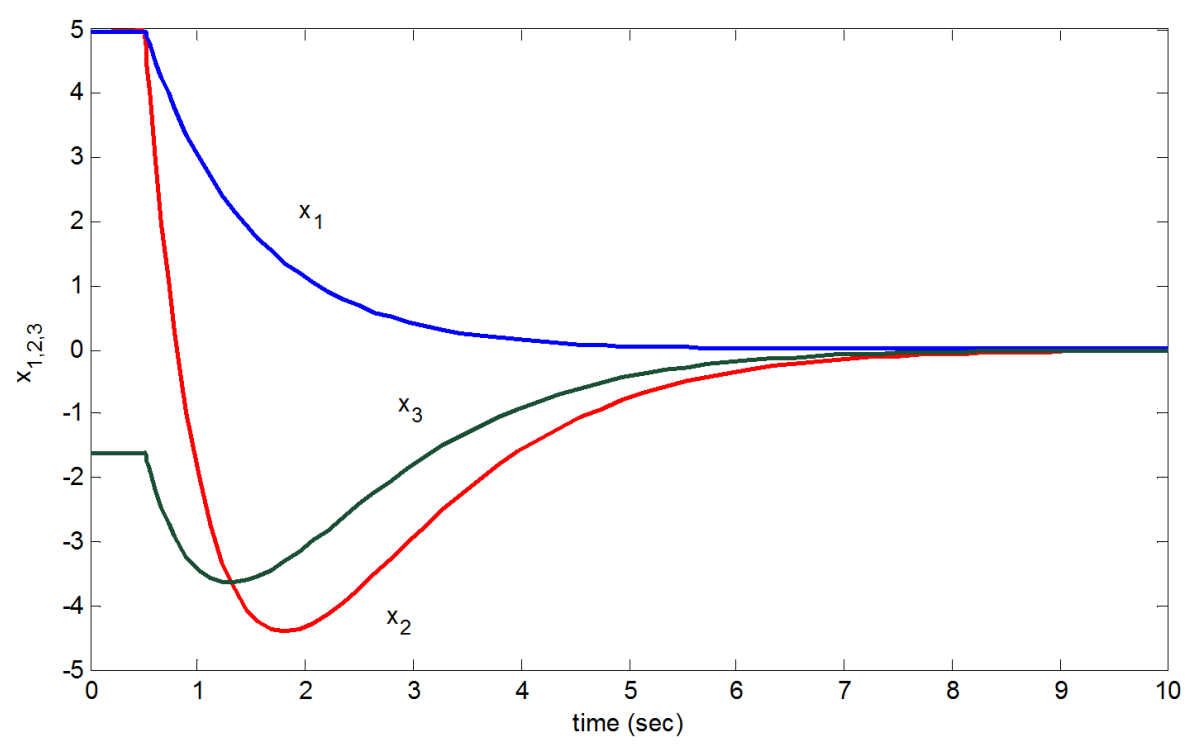

Figure 1: System states' response in the $x$-coordinates.

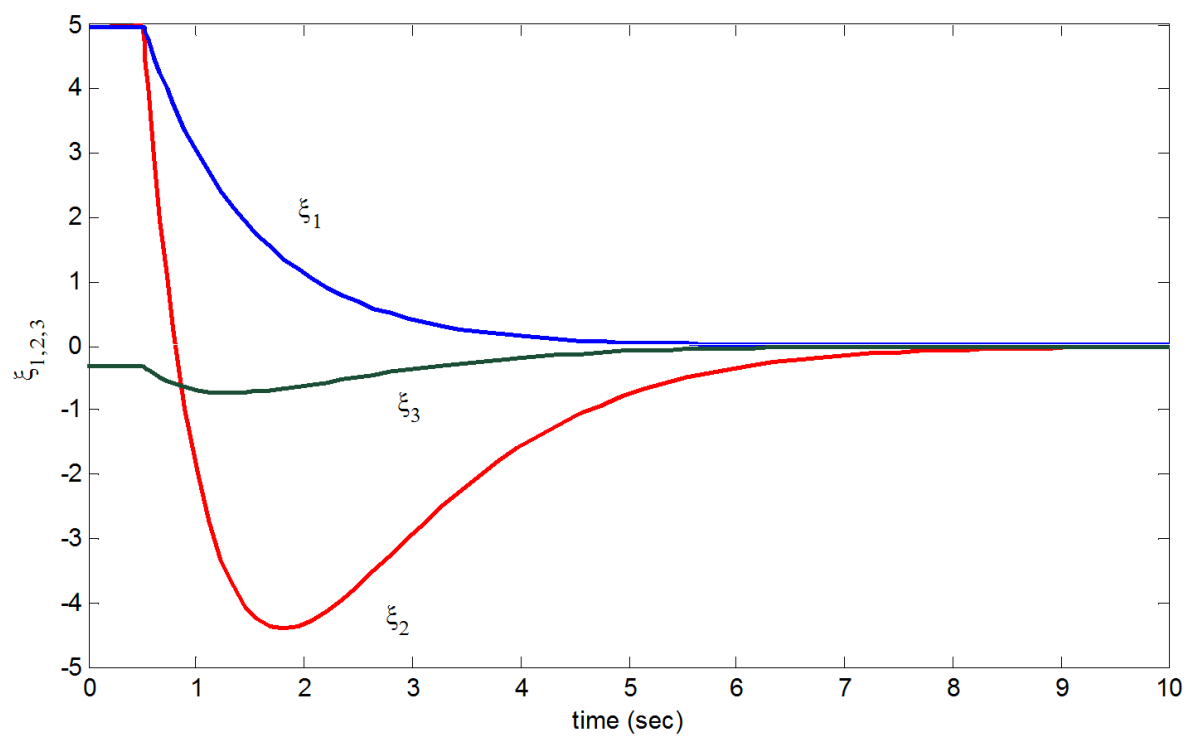

Figure 2: System states' response in $\xi$-coordinates. 


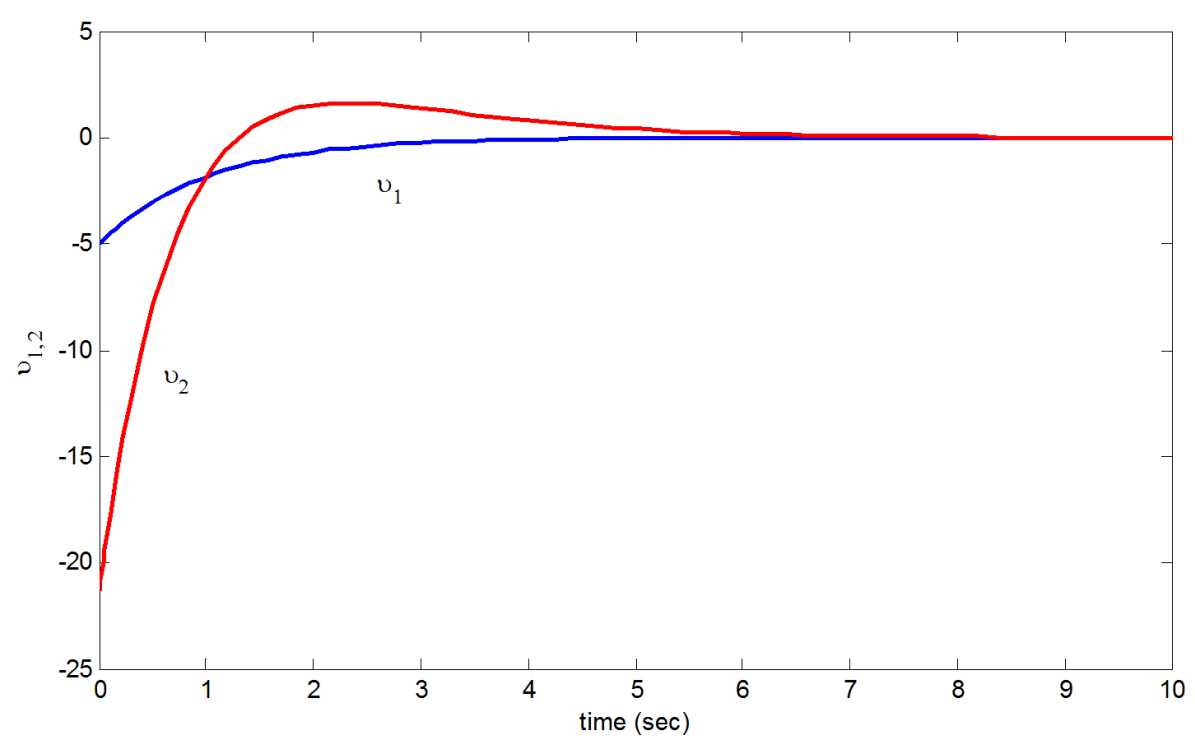

Figure 3: Control inputs.

\section{Conclusion}

In this paper, we addressed the problem of stabilization chained form systems subject to time delay in the input that may be cause by data transmission. The solution we have brought achieves a quasi-exponential convergence for the states of the system. Future research would address random time delays.

\section{References}

[1] R.W. BrocketT: Asymptotic stability and feedback stabilization. In Differential Geometry Control Theory, (R.W. Brockett, R.S. Millman and H.J. Sussmann, eds.), Birkhauser, Boston, 1983, 181-191.

[2] A.M. BLOCH: Nonholonomic Mechanics and Control. Springer, Interdisciplinary Applied Mathematics series, 2015.

[3] Y.G. MARTYNenKo: Motion control of mobile wheeled robots. J. of Mathematical Sciences, 147 (2007), 6569-6606.

[4] A.M. Bloch, M. Reyhanoglu and N.H. McClamroch: Control and stabilization of nonholonomic dynamic systems. IEEE Trans. on Automatic Control, 37(11), (1992), 1746-1757. 
[5] R.T. M'Closkey and R.M. Murray: Nonholonomic systems and exponential convergence: some analysis tools. 32nd IEEE Conf. on Decision and Control, S. Antonio, Texas, (1993), 943-948.

[6] A. Astolfi: Discontinuous control of nonholonomic systems. Systems and Control Letters, 27 (1996), 37-45.

[7] F. MNIF and A.S. AL-YAHMADI: Recursive backstepping stabilization of a wheeled mobile robot. Proc. of the Imech. Part I, J. of Systems and Control Engineering, 219(6), (2006), 419-429.

[8] Y.Q. WU, Y. ZHAO and J.B. YU: Global asymptotic stability controller for uncertain nonholonomic systems. J. of the Franklin Institute, 350(5), (2013), 1248-1263.

[9] E. FRIDMAN: Introduction to Time-Delay Systems. Birkhäuser, Springer, 2014.

[10] P. HokAyem and M. Spong: Bilateral teleoperation: An historical survey. Automatica, 42 (2006), 2035-2057.

[11] N. CHOPRA: Control of robotic manipulators under time varying sensing-control delays. Proc. of the IEEE, Int. Conf. on Robotics and Automation, (2010), 17681773 . 